\title{
QoE-Based Resource Allocation Algorithm for Multi-Applications in Downlink LTE Systems
}

\author{
Peiwen Tang ${ }^{1}$, Ping Wang ${ }^{1}$, Ning Wang ${ }^{1}$, Nguyen Ngoc Van ${ }^{2}$ \\ ${ }^{1}$ Wireless Communication and Multimedia Laboratory, College of Electronic and Information Engineering Tongji University \\ Shanghai, China \\ ${ }^{2}$ School of Electronics and Telecommunications, Hanoi University of Science and Technology, Hanoi, Vietnam. \\ ng_ng_van@yahoo.com, *pwang@tongji.edu.cn
}

\begin{abstract}
This paper proposes a novel approach of cross layer resource allocation based on Quality of Experience (QoE) for multiple applications in downlink Long Term Evolution (LTE) systems. To maximize the level of system QoE while guaranteeing the fairness for users, we introduce a Particle Swarm Optimization (PSO) based resource block allocation algorithm which has a quicker convergence rate. It is much simpler and easier to implement this algorithm with few parameters. Simulations demonstrate that the proposed approach improve the level of the system's QoE more effectively than other algorithms under the circumstance that guarantees user's minimum QoE requirement.
\end{abstract}

Index Terms - PSO, QoE, Resource allocation, LTE

\section{Introduction}

Today, mobile Internet has become an indispensable part of people's life. The function of mobile network includes not only the classic connection in voice, but also video streaming services and data transfer. Though the performance of LTE [1] has been improved a lot than those of the former $2 \mathrm{G}$ and $3 \mathrm{G}$ mobile networks, the mobile access networks are still considered as a bottleneck. To cope with the rise of traffic volume and different requirements of emerging applications, efficient power and sub-carrier allocation technologies [2] are much necessary for LTE system. The traditional methods to ameliorate network performance are based on system Quality of Service (QoS), such as throughput, delay and jitter. However, the requirements of applications are different in terms of network resources. Moreover, QoS can't reflect the end clients' real experience of different applications. Currently, since Quality of Experience (QoE) connects as closely as possible to users' subjective perception, it plays a more important role in indicating users' experience and reaction to certain service or application.

Application-driven cross-layer optimization (CLO) has been studied for many years [3-4], which can jointly optimize APP layer, MAC layer, and PHY layer, but only support single application. In the real scenario, users that share cell wireless resource may run different applications. The impact of losses on the user-perceived quality depends largely on applications. In [5], the authors propose a multiuser cross-layer optimization approach using MOS as performance metric of application layer for multiple applications. To obtain system Max-MOS, it introduces a greedy algorithm to dynamically optimize resource usage of wireless transmission system and improve user perceived experience of services in a multiuser environment. [6] Designs a dynamic optimization algorithm based on the multi-choice knapsack problem (MCKP) for Multi-Service in LTE network which aims at maximizing the user QoE. The utility function considers bitrate and delay factors. [7] Introduces a utility-based application data rate adaptation strategy for QoE-driven resource allocation in a loaded wireless network. It proposes two optimization algorithms, utility maximization algorithm which aims at obtaining the maximal system QoE, and Max-min utility algorithm which considers system fairness as well. In this paper we propose a new resource allocation algorithm for Multi-Service in downlink LTE systems based on the particle swarm optimization (PSO), a population-based optimization algorithm. The proposed algorithm has a quicker convergence rate with simpler implementation.

The rest of this paper is organized as follows: Section II presents the LTE radio link layer model. Section III presents application layer models of four types' applications for CLO. In Section IV, an optimization algorithm based on PSO solving resource allocation problem is presented. Afterwards, the performances of different algorithms are compared in section V. Finally, conclusions are drawn in section VI.

\section{Radio Link Layer Model}

In LTE system, the elementary unit of resources allocation is defined Scheduling Block (SB) while defining one SB as $N_{s}$ consecutive OFDM symbols in the time domain and $N_{s c}$ consecutive subcarriers in the frequency domain. As an elementary unit of LTE resources allocation, one SB as a sub channel should be completely assigned to one user. Define the binary variable $a_{k, n}$ as the resource assignment indicator, with value 1 corresponding to the condition that SB $n$ is allocated to user $k$ and value 0 otherwise, i.e. $\sum_{k=1}^{K} a_{k, n}=1, \forall n$. Define $g_{k, n}$ as channel quality indicator (CQI) of user $k$ on the SB $n$. The eNB can dynamically select the appropriate modulation and coding schemes (MCSs) for each SB according to the fed back $g_{k, n}$. Since frequency unified Adaptive Modulation and Coding (AMC) technology has been used in LTE system, all SBs scheduled for a given user within the same TTI must use the same MCS. Define the binary variable $b_{k, j}$ to indicate the choice of MCS for user $k \cdot b_{k, j}=1$ denotes that MCS $j$ is chosen for user $k$, i.e. 
$\sum_{j=1}^{J} b_{k, j}=1, \forall k$. Due to the existence of pilot and other control signals, only $N_{s c}^{d}(s)$ out of $N_{s c}$ subcarriers can be used to carry date signals in the $s$ th OFDM symbol duration, where $s \in\left\{1,2, \cdots, N_{s}\right\} \quad$ and $\quad N_{s c}^{d}(s) \leq N_{s c}$. In addition, let $R_{j}^{(c)}$ be the code rate associated with MCS $j \in\{1,2, \cdots, J\}$, where $J$ is the total number of MCS supported in the transmission, $M_{j}$ be the constellation size of MCS $j$ and $T_{s}$ be the OFDM symbol duration. Then, the bit rate $r^{(j)}$ achieved by MCS $j$ for a single SB is given by

$$
r^{(j)}=\frac{R_{j}^{(c)} \log _{2}\left(M_{j}\right)}{T_{s} N_{s}} \sum_{s=1}^{N_{s}} N_{s c}^{(d)}(s)
$$

Thus, the data rate achieved by user $k$ can be expressed as

$$
r_{k}=\sum_{n=1}^{N} a_{k, n} \sum_{j=1}^{q_{k, n}} b_{k, j} r^{(j)}
$$

\section{Application Layer Model}

This paper uses the QoE metric to estimate the level of user's satisfaction for the applications. The mean opinion score (MOS) is a possible metric to capture user's satisfaction. Though MOS was originally proposed for voice quality assessment and provided a numerical measure of the quality of the received human voice, it has been extended as a user-perceived quality metric to other services, such as video streaming, web browsing and file download [5-8]. We can map the network and application parameters onto multi-service MOS as below:

$$
\begin{gathered}
Q_{m}=f_{m}(\tilde{x}) \\
f_{m}: R \rightarrow \text { QoEMOS }, m \in\{1,2 \ldots, M\}
\end{gathered}
$$

$\tilde{x}$ is the set of all possible parameter tuples abstracted from the protocol layers that represents a set of candidate distribution modes. Each application is denoted by $m \quad(1 \leq m \leq M)$ while defining different mapping function $f_{m}$ for each application. Then the sum of multi-service MOS for the system is described as follow:

$$
F(\tilde{x})=\sum_{k=1}^{K} \sum_{m=1}^{M} \gamma_{k, m} f_{m}(\tilde{x})
$$

In which user number is denoted by $k(1 \leq k \leq K)$. Define a binary variable $\gamma_{k, m}$, with $\gamma_{k, m}=1$ under the circumstance that user $k$ is running service $m$. Besides, we assume that one user can only run one application, i.e.
$\sum_{m=1}^{M} \gamma_{k, m}=1$. The decision of the optimizer can be expressed as:

$$
\tilde{x}_{\text {opt }}=\arg \max _{\tilde{x} \in X} F(\tilde{x})
$$

Where $\tilde{x}_{\text {opt }}$ is the parameter tuple that maximizes the objective function.

\section{A. VOIP}

The International Telecommunication Union (ITU) has defined E-model to help ensure whether users would be satisfied with the end-to-end transmission performance. The key factor is transmission rating factor $R$, which combines all relevant transmission parameters for the considered connection. This rating factor $R$ is composed of [8]:

$$
R=R o-I s-I d-I e-e f f+A
$$

$R o$ represents the basic signal-to-noise ratio in principle, $I S$ is a combination of all impairments which occur simultaneously with the voice signal, and $A$ allows for compensation of impairment factors. To simplify the calculation, we fit these three non-network factors as a constant $R_{c}$. After lots of experiments, $R_{c}$ may be taken as 93.2. Factor $I d$ represents the impairments caused by delay, and effective equipment impairment factor Ie-eff represents impairments caused by low bit-rate codec and impairments due to packet-losses of random distribution. So it can be sampled as:

$$
R=R_{c}-I d-I e-e f f
$$

Id is further subdivided into the three factors Idte, Idle and Idd.

$$
I d=I d t e+I d l e+I d d
$$

The factor Idte and Idle gives an estimate for the impairments due to Talker Echo and Listener Echo. For simplicity, these two factors are not considered. The factor Idd represents the impairments caused by too long absolute delay $T a$, which occurs even with perfect echo cancelling.

For $T a \leq 100 m s$ :

$$
I d d=0
$$

For $T a>100 m s$ :

$$
\begin{gathered}
I d d=25\left\{\left(1+X^{6}\right)^{\frac{1}{6}}-3\left(1+\left[\frac{X}{3}\right]^{6}\right)^{\frac{1}{6}}+2\right\} \\
X=\log _{2}\left(\frac{T a}{100}\right)
\end{gathered}
$$


Now, the Effective Equipment Impairment Factor Ie-eff which depends on packet-loss is derived using the codec-specific value for the Equipment Impairment Factor at zero packet-loss $I e$ and the Packet-loss Robustness Factor Bpl . BurstR is the so-called Burst Ratio. When packet loss is random (i.e., independent) Burst $R=1$; and when packet loss is bursty (i.e., dependent) BurstR $>1$. Ie-eff is calculated by the following formula:

$$
I e-e f f=I e+(95-I e) \cdot \frac{P p l}{\frac{P p l}{B u r s t R}+B p l}
$$

These parameters are listed in Table I for several codec.

For simplicity, we suppose that packet loss is random, that is BurstR $=1$.

Table I [8]

\begin{tabular}{|c|c|c|c|c|}
\hline $\begin{array}{c}\text { Codec\& Bit } \\
\text { Rate(Kbps) }\end{array}$ & $\begin{array}{c}\text { Codec Sample } \\
\text { Size (Bytes) }\end{array}$ & $\begin{array}{c}\text { Voice Payload } \\
\text { Size (ms) }\end{array}$ & Ie & Bpl \\
\hline G.711(6.3 Kbps) & 80 (Bytes) & $20(\mathrm{~ms})$ & 0 & 25.1 \\
\hline G.729(8 Kbps) & 10 (Bytes) & $20(\mathrm{~ms})$ & 11 & 19.0 \\
\hline G.723.1(64 Kbps) & 24 (Bytes) & $30(\mathrm{~ms})$ & 15 & 16.1 \\
\hline
\end{tabular}

Mapping transmission rating factor $R$ to the users satisfaction metric MOS is the second step in the E-model.

$$
M O S= \begin{cases}1.0 & R<0 \\ 1+0.035 R+R(R-60)(100-R) 7 \cdot 10^{-6} & \text { other } \\ 4.5 & R \geq 100\end{cases}
$$

\section{B. Web browse}

For web browsing, users attach great importance to the page loading speed. Authors in [9] present an analytic function that accurately fits the mean opinion score of their web browsing experimental results.

$$
M O S=5-\frac{578}{1+\left(11.77+\frac{22.61}{d}\right)^{2}}
$$

The factor $d$ represents service response time. We denote $r$ as user data rate, MSS as IP datagram size excluding the TCP/IP header, and RTT as the time taken for a small IP packet to travel from the server to the UE and back. Then the service response time $d$ can be calculated as:

$$
d \approx 3 R T T+\frac{F S}{r}+L \cdot\left[\frac{M S S}{r}+R T T\right]-\frac{2 M S S \cdot\left[2^{L}-1\right]}{r}
$$

The number of slow start cycles with idle periods $L$ cannot be larger than the number of cycles $L_{1}$ the congestion window takes to reach the bandwidth-delay product and the number of slow start cycles $L_{2}$ before the Web page size is completely transferred. So it can be calculated as:

$$
\begin{aligned}
L & =\min \left[L_{1}, L_{2}\right] \\
L_{1} & =\left[\log _{2}\left(\frac{r \cdot R T T}{M S S}+1\right)\right]-1 \\
L_{2} & =\left[\log _{2}\left(\frac{F S}{2 M S S}+1\right)\right]-1
\end{aligned}
$$

In [10], the obtained results show that the lognormal distribution provides a good fitting to the empirical distribution of the web page sizes:

$$
p d f(F S)=\frac{1}{F S \cdot \sigma \cdot \sqrt{2 \pi}} e^{\frac{\ln (F S-\mu)^{2}}{2 \sigma^{2}}}
$$

The parameter $\mu$ is selected to provide an average web page size $310 \mathrm{~KB}$ in the top 1000 of the most popular home pages.

\section{File download}

We assume that the utility of an elastic traffic (e.g., FTP service) is an increasing, strictly concave and continuously different function of throughput. [8] The author takes the logarithmic MOS-throughput relationship to estimate users' satisfaction for file-download applications. MOS is estimated by using current rate $\mathrm{R}$ offered to users and packet error probability PEP:

$$
M O S=a * \log _{10}[b * R *(1-P E P)]
$$

Where $a$ and $b$ are determined from the maximum and minimum user perceived quality. Under the condition that $\mathrm{PEP}=0$, if download speed is less than $20 \mathrm{kbps}$, users almost feel dissatisfied (we define that $M o s=1$ ), and if speed is more than $400 \mathrm{kbps}$, users feel very satisfied (we define that $M o s=4.5$ ). Thus, as coefficients, $a$ and $b$ are set to 3.4011 and 0.0984 respectively.

\section{Stream video}

Peak signal-to-noise ratio (PSNR) is an objective measurement of video quality which is widely used due to its simplicity and high degree of correlation with subjective quality. However, [11] show that such pixel-based distortion measure does not match well to user perceived visual quality due to the fact that human eyes are highly adapted to structural information. In [12], authors first classify the video content from raw video by extracting the spatial and temporal features. This is accomplished by using a well-known multivariate statistical analysis, called cluster analysis, which can group samples that have various characteristics into similar groups. Then adjust the prediction model according to different classification.

1) Group 1 is classified as 'Slight Movement' (SM), which includes sequences with a small moving region of interest (face) on a static background. 
2) Group 2 is classified as 'Gentle Walking' (GW), which includes wide-angled clips in which both background and content is moving.

3) Group3 is classified as 'Rapid Movement' (RM), which includes sports type of video clips.

The prediction model is obtained by nonlinear analysis of the QoS parameters both in the application and network layer and is given as below.

$$
M O S=\frac{a_{1}+a_{2} F R+a_{3} \ln (S B R)}{1+a_{4} P E P+a_{5}(P E P)^{2}}
$$

Where SBR denotes Sender Bite rate, FR denotes Frame Rate and PEP denotes Packet Error probability. As the re-fitted metric coefficients a1, a2, a3, a4 and a5 along with $R^{2}$ show the goodness of fit for all three video groups in Table II.

Table II [12]

\begin{tabular}{|c|c|c|c|}
\hline Coeff & SM & GW & RM \\
\hline $\mathrm{a} 1$ & 2.797 & 2.273 & -0.0228 \\
\hline $\mathrm{a} 2$ & -0.0065 & -0.0022 & -0.0065 \\
\hline $\mathrm{a} 3$ & 0.2498 & 0.3322 & 0.6582 \\
\hline $\mathrm{a} 4$ & 2.2073 & 2.4984 & 10.0437 \\
\hline $\mathrm{a} 5$ & 7.1773 & -3.7433 & 0.6865 \\
\hline
\end{tabular}

\section{QoE-Driven Resource Block Allocation Algorithm for Multi-Service}

Based on the MOS framework described in section III, we can optimize the system by taking actual user perceived quality of service into account. Except for maximizing the sum of all users' MOSs, we also consider users' fairness. To maximize the system QoE, we propose a QoE-driven resource block allocation algorithm based on the standard Particle Swarm Optimization (PSO) described in [13] for multi-service in LTE-Advanced system.

\section{A. Particle coordinate mapping}

Step one is to map all resource block schemes onto a group of search space particles. Each particle represents a scheme, and its coordinate $x_{i}^{t}=\left(x_{i 1}^{t}, x_{i 2}^{t}, \cdots, x_{i d}^{t}, x_{i D}^{t}\right)^{T}$ stands for the scheme's dimensions. One SB only can be allocated to one user. If the system has $D$ SBs and $K$ users, SB's chosen user must be $[1, \mathrm{k}]$. In order to map a resource block allocation scheme into a particle coordinate, we can let the scale in the $d$ th coordinate dimension represent user's MOS rate selected by the scheme in the $d$ th SB. So the particle coordinate has $D$ dimensions, and each dimension's value is an integer from 1 to $K$. At the same time, we give every particle a random velocity vector $v_{i}^{t}=\left(v_{i 1}^{t}, v_{i 2}^{t}, \cdots, v_{i d}^{t}, v_{i D}^{t}\right)^{T}$, which stands for the particle developing direction.

\section{B. Fitness function}

In step two, a fitness function is defined to evaluate the quality of the schemes. With the objective of maximizing the sum of all users' MOSs (Max-MOS), the optimization problem can be modeled as

$$
\text { Fitness }=\sum_{k=1}^{K} \sum_{m=1}^{M} \gamma_{k, m} \cdot \operatorname{Mos}_{k}
$$

Where $\gamma_{k, m}$ denotes that user $K$ is running service $\mathrm{m}$ and we assume that one user can only run one application, i.e. $\sum_{m=1}^{M} \gamma_{k, m}=1$. But in some condition, a user with good channel condition may get excellent QoE, that is, MOS is more than 4.0. And user with bad channel condition can only get MOS less than 1.0. Such condition is thought unfair. Thus, we construct the penalty function as

$$
\text { Penalty }=\sum_{k=1}^{K}\left[\min \left(0, \operatorname{Mos}_{k}-3\right)\right]^{2}
$$

It means that if any users' MOS is less than 3, the whole system's QoE will be punished. It can guide particle moving to "fairer" position. So the final fitness function is given as below

$$
\text { Fitness }=\sum_{k=1}^{K} \sum_{m=1}^{M} \gamma_{k, m} \cdot \operatorname{Mos}_{k}-\sum_{k=1}^{K}\left[\min \left(0, \operatorname{Mos}_{k}-3\right)\right]^{2}
$$

\section{Particle coordinate update}

In step three, each particle will update its velocity and coordinate vector referring to its historical personal best position $p_{i}^{t}=\left(p_{i 1}^{t}, p_{i 2}^{t}, \cdots, p_{i d}^{t}, p_{i D}^{t}\right)^{T}$ and global best position $p_{g}^{t}=\left(p_{g 1}^{t}, p_{g 2}^{t}, \cdots, p_{g d}^{t}, p_{g D}^{t}\right)^{T}$. The update function is given as below.

$$
\begin{gathered}
v_{i d}^{t+1}=w v_{i d}^{t}+\operatorname{rand}_{1} c_{1}\left(p_{i d}^{t}-x_{i d}^{t}\right)+\operatorname{rand}_{2} c_{2}\left(p_{g d}^{t}-x_{i d}^{t}\right) \\
x_{i d}^{t+1}=x_{i d}^{t}+v_{i d}^{t+1}
\end{gathered}
$$

Where $w$ is an inertia weight. $r a n d_{1}$ and $r a n d_{1}$ are the random number following the uniform distribution between [0, 1], and $c_{1}$ and $c_{2}$ are two learning factors. These make every particle have ability to learn from group's excellent particle position $p_{g d}^{t}$ and its history excellent position $p_{i d}^{t}$, in order to move closer to a more outstanding position. In the search process, the particles remember their experience while making reference to the experience of their peers. As the standard PSO is designed for a continuous solution space, we introduce an operation, that denoted by INT, to discretize position and velocity of particles [13]. 


$$
M=I N T(r)=\left\{\begin{array}{l}
\text { floor }(r) \text { if rand }>r-\text { floor }(r) \\
\text { ceil }(r) \text { otherwise }
\end{array}\right.
$$

For the integer $\mathrm{M}$, we first search it as a continuous variable $r$. Then select the integer value randomly from floor $(r)$ and ceil $(r)$ with the probability in inverse proportional to their distance, which can enhance the local search ability of individuals in comparison with a determinant method such as round-off operation. So after discretization, the velocity update function can be expressed as follows

$$
v_{i d}^{t+1}=\operatorname{INT}\left(w v_{i d}^{t}+\operatorname{rand}_{1} c_{1}\left(p_{i d}^{t}-x_{i d}^{t}\right)+\operatorname{rand}_{2} c_{2}\left(p_{g d}^{t}-x_{i d}^{t}\right)\right)
$$

In every iteration, check whether the particles have reach the scheduled termination criterion. If satisfied, then output; otherwise, enter the next iteration.

\section{Simulation Results}

In this section, the proposed PSO algorithm is compared with other two allocation algorithms: maximum throughput algorithm (Max throughput) in [14] and the maximizing sum MOS (Max-MOS) algorithm described in Section IV. In the simulation, we assume six types of services are running in the network: three types of videos mentioned in Tab. II as the test video resources and other three types of services described in Section III. The number of users is increased from 6 to 24 . In the system we assume $N=28 \mathrm{SBs}$ per Transmission Time Interval (TTI); $\mathrm{SB}$ is constructed as $N_{s}(=14)$ consecutive OFDM symbols in the time domain and $N_{s c}(=12)$ consecutive subcarriers in the frequency domain. Moreover, the parameters of PSO algorithm are set as follows: the number of particles $M(=30)$, the maximum number of iterations is 100 , the learning factors $c_{1}=c_{2}=2$, the inertia weight $w$ decreases linearly from 0.9 to 0.4 .

Fig. 1 and Fig. 2 show the means and standard deviations (STDs) of multi-service MOSs of different algorithms with user number increasing from 6 to 24. It can be seen that the mean MOS of Max throughput is the worst in the three algorithms, as it doesn't take QoE parameters and service fairness into consideration. The mean MOS value of Max throughput's declines rapidly while the users number increase. The mean MOS values obtained from the two PSO based algorithms are nearly the same and both are much higher than that obtained from the Max throughput algorithm. Because of considering service fairness, the proposed algorithm sacrificed a little system QoE comparing with Max-MOS. From Fig.2, we can see that when user number is less than 12 , the two PSO based algorithms both obtain low STDs and make every user's MOS higher than 4.2 because of the sufficient system resource. However, when user number increasing, the proposed algorithm attains the smaller STDs than Max-Mos does. That is, the proposed algorithm achieves better fairness among services.

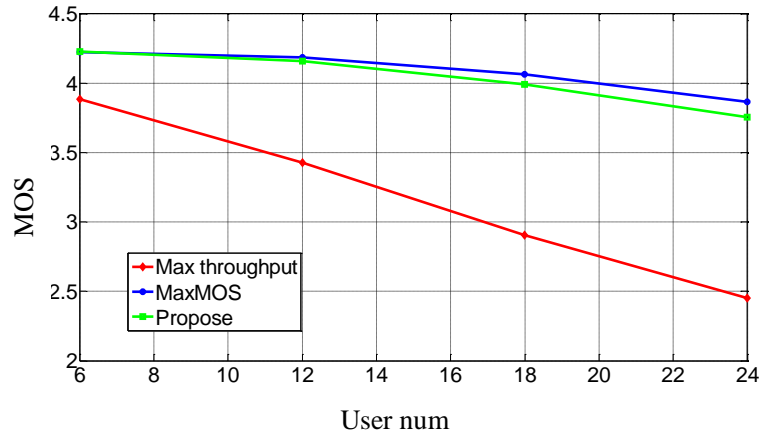

Fig.1. system mean MOSs versus the number of users

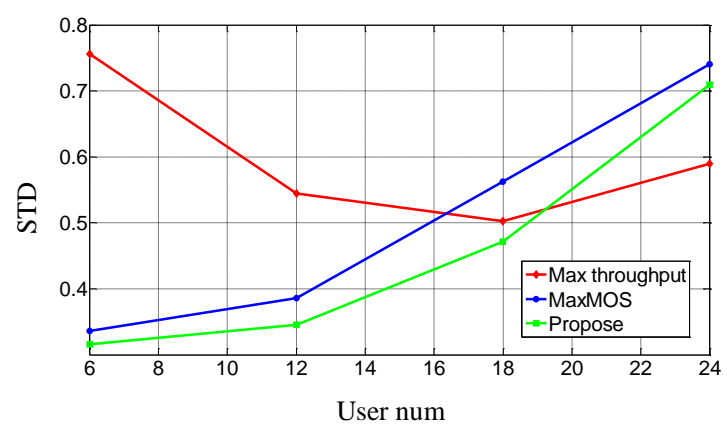

Fig.2. system MOS standard deviations versus the number of users

The performances of the mean MOS values with different services are compared in Fig.3. The MAX throughput algorithm aims to maximize the system throughput without considering user's QoE. Thus, it performs worst in all types of services. From Fig.3 we can see that RM service takes up the most system resources to get the same MOS comparing with other services. In the Max-MOS algorithm, RM service's mean MOS is only 2.88. As the proposed algorithm guides search particles moving to "fairer" position, the RM service's mean MOS is 3.07. Therefore, the proposed algorithm could sacrifice the system QoE to make every user attain basic QoE requirements.

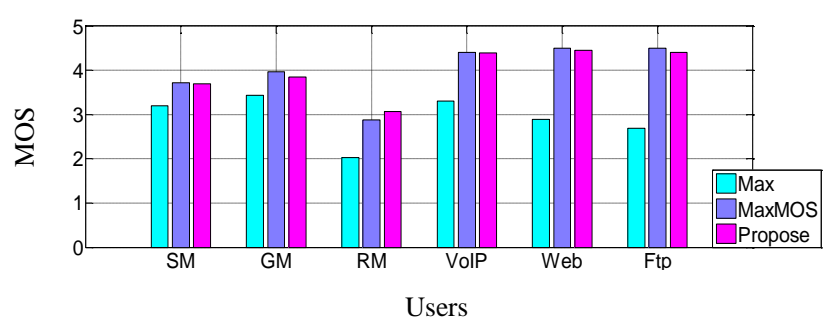

Fig.3. mean MOSs of various applications in different algorithms

Fig. 4 illustrates SB allocation status of the two PSO based algorithms with 18 and 24 users. It can be seen that Max-MOS algorithm inclines to allocate SB to Web and Ftp service, while the proposed algorithm inclines to allocate SB to video streaming services which demand higher transmission rate to keep system "fairness". Especially in the case of 24 users, the RM users get the most SBs among all users. 


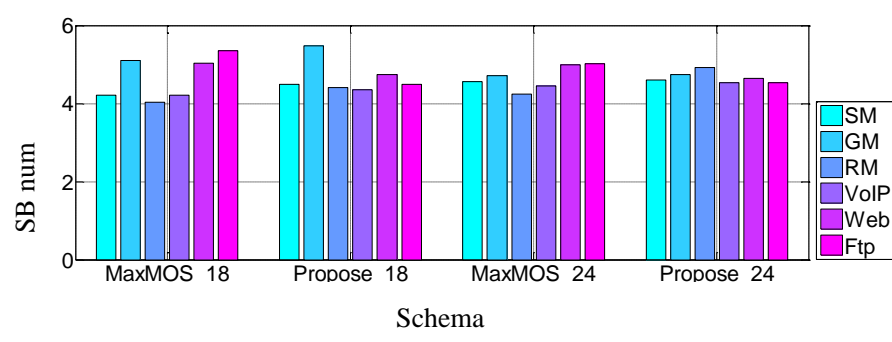

Fig.4. The SB configurations of various applications with 18 and 24 users

\section{Conclusions}

In this paper, a novel QoE-based resource block allocation algorithm is proposed for multi-application in LTE-Advanced networks. PSO has been applied to the proposed algorithm which maps SB allocation schemes onto particles; updates the particle's position and velocity learning from group's excellent particle position and its history excellent position; iterates several times to attain the optimal allocation scheme. The simulation results show that the two PSO-based algorithms achieve a much better user perceived quality compared with the throughput maximization algorithm. On the other hand, since we introduced the penalty factor, the proposed algorithm can guarantee users' minimum QoE requirement via fair allocation of system resource.

\section{Acknowledgment}

This work was supported by a grant from National High Technology Research and Development Program of China (863 Program) (2012AA111902) the Fundamental Research Funds for the Central Universities (0800219162).

\section{References}

[1] D. Astely and E. Dahlman, "LTE: the evolution of mobile broadband," Communications Magazine, vol. 47, no. 3, pp. 44-51, 2009.
[2] G. Wunder, C. Zhou, H.-E. Bakker, and S. Kaminsk, "Thoughput Maximization under Rate Requirements for the OFDMA Downlink Channel with Limited Feedback," EURASIP Journal on Wireless Communications and Networking- Multicarrier Systems, January 2008.

[3] S. Khan, Y. Peng, E. Steinbach, M. Sgroi, and W. Kellerer, "Application-driven cross-layer optimization for video streaming over wireless networks," IEEE Communications Magazine, vol. 44, no. 1, pp. 122-130, 2006.

[4] M. van der Schaar and S. Shankar N., "Cross-layer wireless multimedia transmission: challenges, principles, and new paradigms," IEEE Wireless Communications, vol. 12, no. 4, pp. 50-58, 2005.

[5] S. Khan, S. Duhovnikov, et al, "MOS-based multiuser multiapplication cross-layer optimization for mobile multimedia communication," Advances in Multimedia, Article ID 94918, 2007.

[6] Fei Liu, Wei Xiang, Yueying Zhang, Kan Zheng, Hui Zhao, “A Novel QoE-Based Carrier Scheduling Scheme in LTE-Advanced Networks with Multi-Service," Vehicular Technology Conference (VTC Fall), 2012 IEEE pp. 1-5, 2012.

[7] Thakolsri, S. ; Kellerer, W. ; Steinbach, E. "Application-Driven Cross Layer Optimization for Wireless Networks using MOS-based Utility Functions," Communications and Networking in China, 2009. ChinaCOM 2009. Fourth International Conference on , pp. 1-5, 2009

[8] ITU-T Rec. G.107, "The E-model, a computational model for use in transmission planning," (2005-03), available www.itu.int

[9] P. Ameigeiras, J. J. Ramos-Munoz, et al, "QoE Oriented Cross-Layer Design Of a Resource Allocation Algorithm In Beyond 3G systems," Computer Communications, vol. 33, no. 5, pp. 571-582, 2010.

[10] M. Molina et al., "Web traffic modeling exploiting TCP connections' temporal clustering through HTML-REDUCE Network," IEEE 14 (3) (2000) 46-55.

[11] Z. Wang, A. C. Bovik, H. R. Sheikh, and E. P. Simoncelli, "Image quality assessment: From error visibility to structural similarity," IEEE Transactions on Image Processing, vol. 13, no. 4, pp. 600612, Apr. 2004.

[12] A. Khan, L. Sun, E. Jammeh, and E. Ifeachor, "Quality of experience driven adaptation scheme for video applications over wireless networks," IET Communications, vol. 4, no. 11, pp. 1337-1347, 2010.

[13] D.M. Lei, "A Pareto archive particle swarm optimization for multi-objective job shop scheduling," Computers \& Industrial Engineering, 2008, 54 (4): 960-971.

[14] Raymond Kwan, Cyril Leung, and Jie Zhang, "Resource Allocation in an LTE Cellular Communication System," IEEE International Conference on Communications, 2009. 